J. Math. Kyoto Univ. (JMKYAZ)

13-1 (1973) 171-190

\title{
Simple groups of conjugate type rank 5
}

\author{
By \\ Noboru Iто* \\ (Communicated by Professor Nagata, August 4, 1972)
}

\section{Introduction}

Let $(S)$ be a finite group, $I(B)$ the set of indices of centralizers of non-central elements of $(S)$ in $(B)$, and $r$ the number of elements in $I$ (B).$\quad r$ is called the conjugate type rank of $\mathbb{S}$. We introduce an ordering in $I$ (SB) as follows:let a and $b$ be two elements of $I$ (S)). Then $a>b$ if and only if a divides $b$. Let $k$ be the number of maximal elements in $I(B)$. Then (S) is called k-headed. We form a graph $C(\mathbb{B S})$ of $(B)$ as follows: the points of $C(\mathbb{S})$ are the elements of $I$ (B)). The (oriented) edge ab of $C$ (B) exists, where a and $b$ are points of $C(\mathbb{S})$, if and only if $a>b$. We denote the edge ab by a. $C(\&)$ is called the conjugate type graph of (S). The centralizer $\stackrel{\uparrow}{b}$

of any non-central element of (S) in (S) corresponding to an isolated point of $C$ (S) is called free.

An obvious problem is as follows: Let $r$ be a given positive integer. Then classify all (simple) groups (3) such that conjugate type rank of (S) are equal to $r$. When $r$ increases, this problem probably will become more difficult with exponential growth rate. If, however, the shape of $C(\mathbb{S})$ is given and coincident with that of the conjugate type graph of some known simple group, then the problem will become considerably tractable.

In previous papers we proved the following theorems:

(I) [7] A finite group $(S)$ is a simple group of the conjugate type

\footnotetext{
* This work is partially supported by NSF GP 28420 .
} 
rank 3 if and only if $(S)$ is isomorphic with some $L F\left(2,2^{m}\right), m \geqq 2$.

(II) [8] A finite group (S) is a simple group of the conjugate type rank 4 if and only if $(S)$ is isomorphic with some $\operatorname{LF}(2, q)$, where $q \geqq 7$ is odd.

It the present paper we prove the following theorem:

Theorem. A simple group of conjugate type rank 5 and not of 3-headed is isomorphic with some $S z(l), l=2^{2 n+1}, n \geqq 1$, or $\operatorname{LF}(3,4)$.

Remark. The 3-headed case is still open.

Notation and definition. Let $\mathfrak{X}$ be a finite group. $\boldsymbol{Z}(\mathfrak{X})$ is the center of $\mathfrak{X}$. If $\mathfrak{X}$ is solvable, then $\boldsymbol{F}(\mathfrak{X})$ is the Fitting subgroup of $\mathfrak{X}$. Let $\mathfrak{Y}$ be a subset of $\mathfrak{X}$. $|\mathfrak{Y}|$ is the number of elements in $\mathfrak{Y}$. $\pi(\mathfrak{X})$ is the set of prime divisors of $|\mathfrak{X}|$. If $\mathfrak{Y}$ is nonempty, then $\boldsymbol{C s} \mathfrak{Y}$ is the centralizer of $\mathfrak{Y}$ in $\mathfrak{X}$. If $\mathfrak{Y}=\{Y\}, \boldsymbol{C s} \mathfrak{Y}=\boldsymbol{C s} Y . \quad \boldsymbol{N} \boldsymbol{s} \mathfrak{Y}$ is the normalizer of $\mathfrak{Y}$ in $\mathfrak{X}$. $\langle\mathfrak{V}\rangle$ is the subgroup generated by $\mathfrak{Y}$. If $\mathfrak{Y}=\{Y\},\langle\mathfrak{Y}\rangle=\langle Y\rangle$. Let $\mathbb{Z}$ be a subset of $\mathfrak{X}$. Then [Y), 3$]$ is the subset of $\mathfrak{X}$ consisting of $Y^{-1} Z^{-1} Y Z$, where $Y$ and $Z$ are elements of $\mathfrak{Y}$ and $\mathfrak{Z}$, respectively. $A$ proper subgroup $\mathfrak{F}$ of $\mathfrak{X}$ is called fundamental, if there exists an element $X$ of $\mathfrak{X}$ such that $\mathfrak{F}=\boldsymbol{C s} X$. $\mathfrak{F}$ is called maximal, if $\mathfrak{F}$ is containd in no other fundamental subgroups of $\mathfrak{X}$. $\mathfrak{F}$ is called minimal, if $\mathfrak{F}$ contains no other fundamental subgroup of $\mathfrak{X}$. $\mathfrak{F}$ is free, if $\mathfrak{F}$ is maximal and minimal.

\section{2-headed case}

The purpose of this section is to show that this case does not occur.

Let $(S)$ be a simple group of conjugate type rank 5 and of 2 headed. Let $n_{i}$ be maximal elements of $I(\mathbb{S})(i=1,2)$. Let $A_{i}$ be an element of (B) such that (S) : $\boldsymbol{C s} A_{i}=n_{i}(i=1,2)$. Then the class equation implies that $\left(n_{1}, n_{2}\right)=1$. In particular, $\mathscr{S}=\boldsymbol{C} \boldsymbol{s} A_{1} \boldsymbol{C s} A_{2}$.

(2.1) Both $\boldsymbol{C s} A_{1}$ and $\boldsymbol{C s} A_{2}$ arc not free.

Proof. See the proof of (2.2) in [8]. 
(2.2) We may assume that $\left|\left\langle A_{i}\right\rangle\right|=p_{i}$ is a prime $(i=1,2)$.

Then $p_{1} \neq p_{2}, A_{i}$ is $p_{i}$-central, namely $A_{i}$ belongs to the center of some Sylow $p_{i}$-subgroup of $\mathbb{E}, n_{2} \equiv 0\left(\bmod p_{1}\right)$ and $n_{1} \equiv 0\left(\bmod p_{2}\right)$.

Proof. See the proof of (2.3) in [8].

(2.3) We have that either $\left|\boldsymbol{C s} A_{1}\right| \not \equiv 0\left(\bmod p_{2}\right)$ or $\left|\boldsymbol{C} \boldsymbol{s} A_{2}\right| \not \equiv 0$ $\left(\bmod p_{1}\right)$.

Proof. Assume the contrary that both $\left|\boldsymbol{C s} A_{1}\right| \equiv 0\left(\bmod p_{2}\right)$ and $\left|\boldsymbol{C} \boldsymbol{s} A_{2}\right| \equiv 0\left(\bmod p_{1}\right)$.

Let $A_{2}^{\prime}(\neq E)$ be an element of the center of a Sylow $p_{2}$-subgroup of $\boldsymbol{C} \boldsymbol{s} A_{1}$. We may assume that $A_{2}^{\prime}$ belongs to $\boldsymbol{C} \boldsymbol{s} A_{2}$. If $\left|\boldsymbol{C} \boldsymbol{s} A_{2}^{\prime}\right|=$ $\left|\boldsymbol{C} \boldsymbol{s} A_{2}\right|$, then $\mathbb{B}=\boldsymbol{C} \boldsymbol{s} A_{1} \boldsymbol{C s} A_{2}^{\prime}$. Since $A_{1} A_{2}^{\prime}=A_{2}^{\prime} A_{1}$, this implies that $\mathbb{B}$ is not simple. If $\left|\boldsymbol{C} \boldsymbol{s} A_{2}^{\prime}\right|=\left|\boldsymbol{C} \boldsymbol{s} A_{1}\right|$, then $\mathbb{B}=\boldsymbol{C} \boldsymbol{s} A_{2}^{\prime} \boldsymbol{C} \boldsymbol{s} A_{2}$. Since $A_{2}^{\prime} A_{2}=$ $A_{2} A_{2}^{\prime}$, this implies that $(B)$ is not simple. If $\boldsymbol{C s} A_{1} A_{2}^{\prime}=\boldsymbol{C} \boldsymbol{s} A_{2}^{\prime}$, then $A_{1}$ belongs to $\boldsymbol{Z}\left(\boldsymbol{C} \boldsymbol{s} A_{2}^{\prime}\right)$. Hence $A_{1} A_{2}=A_{2} A_{1}$. Then (B) is not simple.

Now $\boldsymbol{C} \boldsymbol{s} A_{1}: \boldsymbol{C s} A_{1} A_{2}^{\prime}$ is prime to $p_{2}$. Let $\mathfrak{P}_{2}$ be a Sylow $p_{2}$-subgroup of $\boldsymbol{C s} A_{2}$. Then we may assume that $\boldsymbol{C s} A_{2}^{\prime}$ contains $\boldsymbol{Z}\left(\Re_{2}\right)$. Since we may assume that $A_{2}$ does not belong to $\boldsymbol{C s} A_{1}$, we may assume that $\boldsymbol{C} \boldsymbol{s} A_{1} A_{2}^{\prime}$ contains no conjugates of $\boldsymbol{Z}\left(\mathfrak{P}_{2}\right)$. Thus we have that $\boldsymbol{C} \boldsymbol{s} A_{2}^{\prime}: \boldsymbol{C} \boldsymbol{s} A_{1} A_{2}^{\prime} \equiv 0\left(\bmod p_{2}\right)$. Hence $\left|\boldsymbol{C} \boldsymbol{s} A_{2}^{\prime}\right|$ does not divide $\left|\boldsymbol{C} \boldsymbol{s} A_{1}\right|$, but $\left|\boldsymbol{C s} A_{2}^{\prime}\right|$ is a proper divisor of $\left|\boldsymbol{C s} A_{2}\right|$. Therefore a part of $C$ (\$) has the shape $n_{1} n_{2}$. Now by symmetry we can conclude that $\stackrel{\uparrow}{\vdots} \uparrow$

$C$ (B) has the shape $n_{1} n_{2}$.

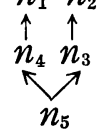

Now assume that there exists a prime divisor $q$ of $|\mathbb{B}|$ such that $q$ is prime to $n_{5}$. Then for every element $X$ of \&s $\boldsymbol{C s} X$ contains a Sylow $q$-subgroup $\mathfrak{Q} \neq \mathbb{F}$ of $\mathbb{B}$. Hence $\boldsymbol{C} \mathfrak{s} \mathfrak{Q}$ and its conjugates exhaust (B). This implies that $\mathbb{B}=\boldsymbol{C} \boldsymbol{s} \mathfrak{Q}$. Hence $(\mathcal{B}$ is not simple. By a theorem of Burnside [5, p. 451] $n_{1}$ is not a prime power. Let $p_{2}^{*}$ be a prime divisor of $n_{1}$ distinct from $p_{2}$. Let $\mathfrak{B}_{2}^{*}$ be a Sylow 
$p_{2}^{*}$-subgroup of (S) contained in $\boldsymbol{C s} A_{2}$. Now assume that (B) : $\boldsymbol{C s} Z \neq n_{2}$ for every element $Z \neq E$ of $Z\left(\mathfrak{B}_{2}^{*}\right)$. Let $A_{2}^{*} \neq E$ be an element of $\boldsymbol{Z}\left(\mathfrak{P}_{2}^{*}\right)$. Then $\boldsymbol{C s} A_{2}^{*}=\boldsymbol{C} \boldsymbol{s} A_{2} A_{2}^{*}$. Thus (S): $\boldsymbol{C s} A_{2}^{*}=n_{4}$. On the other hand, we may assume that $\boldsymbol{C s} A_{2}^{\prime}$ contains $\mathfrak{P}_{2}^{*}$. Otherwise, replace $A_{2}^{*}$ and $A_{2}$ by their appropriate conjugates. Then $\boldsymbol{C} s A_{2}^{\prime}=\boldsymbol{C s} A_{2}^{\prime} A_{2}^{*}$. Thus $\boldsymbol{C s} A_{2}^{\prime}=\boldsymbol{C} \boldsymbol{s} A_{2}^{*}$. Since $A_{2}$ belongs to $\boldsymbol{Z}\left(\boldsymbol{C} \boldsymbol{s} A_{2}^{*}\right)$ and since $A_{1}$ belongs to $\operatorname{Cs} A_{2}^{\prime}, A_{1} A_{2}=A_{2} A_{1}$. Hence (S) is not simple. Thus there exists a $p_{2}^{*}$-element $A_{2}^{*} \neq E$ such that $\boldsymbol{C s} A_{2}=\boldsymbol{C} \boldsymbol{s} A_{2}^{*}$.

Now clearly $\left|\boldsymbol{C s} A_{1}\right| \equiv 0\left(\bmod p_{2}^{*}\right)$. Arguing with $p_{2}^{*}$ instead of $p_{2}$, we obtain that $n_{5} / n_{1}$ is prime to $p_{2}^{*}$ and that $n_{5} / n_{4}$ is divisible by $p_{2}^{*}$. Let $A_{2}^{* \prime} \neq E$ be a $p_{2}^{*}$-element of $\boldsymbol{C} s A_{1} A_{2}^{\prime}$. Then we may assume that $\boldsymbol{C} \boldsymbol{s} A_{1} A_{2}^{\prime}=\boldsymbol{C} \boldsymbol{s} A_{1} A_{2}^{* \prime}$. Hence, since $\boldsymbol{C} \boldsymbol{s} A_{1} A_{2}^{\prime}$ is minimal, $\boldsymbol{C s} A_{1} A_{2}^{\prime}$ is nilpotent. Let $\mathfrak{F}_{2}^{* \prime}$ be a Sylow $p_{2}^{*}$-subgroup of $\boldsymbol{C s} A_{2}^{\prime}$. Let $A_{2}^{* \prime \prime} \neq E$ be an element of $\boldsymbol{Z}\left(\mathfrak{B}_{2}^{* \prime}\right)$. Then $\boldsymbol{C} \boldsymbol{s} A_{2}^{\prime}=\boldsymbol{C} \boldsymbol{s} A_{2}^{\prime} A_{2}^{* \prime \prime}$. Thus $A_{2}^{* \prime \prime} A_{1}=A_{1} A_{2}^{* \prime \prime}$ and $A_{2}^{* \prime \prime}$ belongs to $\operatorname{Cs} A_{1} A_{2}^{\prime}$. If $\left|\boldsymbol{C s} A_{2}^{* \prime \prime}\right|=\left|\boldsymbol{C s} A_{2}\right|$ then (5) is not simple. We may assume that $\left|\boldsymbol{C} \boldsymbol{s} A_{2}^{* \prime \prime}\right|=\left|\boldsymbol{C s} A_{2}^{\prime}\right|$. If $\boldsymbol{C s} A_{2}^{\prime}$ is not maximal, there exists an element $A \neq E$ of (S) such that $|\boldsymbol{C s} A|=\left|\boldsymbol{C s} A_{2}\right|$ and $A A_{1}=A_{1} A$. Then (S) is not simple. So we may assume that $\boldsymbol{C s} A_{2}^{\prime}$ is maximal. Now in the theorem of Camina [2] we may put $\pi=\pi\left(\boldsymbol{Z}\left(\boldsymbol{C s} A_{2}^{\prime}\right)\right)$. Then since $\pi$ contains at least two prime numbers we obtain that $\boldsymbol{C s} A_{2}^{\prime}$ is nilpotent. Then clearly $A_{1} A_{2}$ $=A_{2} A_{1}$. Thus (3) is not simple.

(2.4) We have that both $\left|\boldsymbol{C s} A_{1}\right| \not \equiv 0\left(\bmod p_{2}\right)$ and $\left|\boldsymbol{C} \boldsymbol{s} A_{2}\right| \not \equiv 0$ $\left(\bmod p_{1}\right)$.

Proof. Assume that $\left|\boldsymbol{C s} A_{1}\right| \equiv 0\left(\bmod p_{2}\right)$. Then by (2.3) $\left|\boldsymbol{C s} A_{2}\right| \not \equiv 0\left(\bmod p_{1}\right)$. Let $A_{2}^{\prime} \neq E$ be an element of the center of a Sylow $p_{2}$-subgroup of $\boldsymbol{C s} A_{1}$. Then as in the beginning of the proof of (2.3) we obtain that $\left|\boldsymbol{C s} A_{2}^{\prime}\right| \neq\left|\boldsymbol{C s} A_{1}\right|,\left|\boldsymbol{C} \boldsymbol{s} A_{2}\right|$ and that $\boldsymbol{C s} A_{2}^{\prime} \neq$ $\operatorname{Cs} A_{1} A_{2}^{\prime}$. Anyway $\left|\boldsymbol{C s} A_{1} A_{2}^{\prime}\right| \equiv 0\left(\bmod p_{1}\right)$. Further we see that as in the second part of the proof of (2.3) $\left|\boldsymbol{C s} A_{2}^{\prime}\right| \operatorname{divides}\left|\boldsymbol{C s} A_{2}\right|$. This is a contradiction. 
(2.5) $\boldsymbol{C s} A_{1}$ and $\boldsymbol{C s} A_{2}$ are Hall subgroups of (s.

Proof. See the proof of (2.7) in [8].

Now we see that $C(\mathbb{B S})$ has either the shape $\begin{array}{r}n_{1} \\ n_{3} n_{4}\end{array} n_{5}$ or the

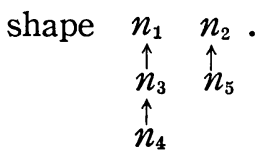

(2.6) $\quad \boldsymbol{C s} A_{2}$ is not nilpotent.

Proof. Assume that $\boldsymbol{C s} A_{2}$ is nilpotent. Since $\boldsymbol{C s} A_{2}$ is not free, Cs $A_{2}$ is obviously not abelian. We may assume that the Sylow $p_{2}-$ subgroup $\mathfrak{P}_{2}$ of $\boldsymbol{C s} A_{2}$ is not abelian. Then the Sylow $p_{2}$-complement $\mathfrak{U}$ of $\boldsymbol{C s} A_{2}$ is abelian. By a theorem of Burnside [5, p. 491] $\mathfrak{u} \neq \mathfrak{F}$. Let $X \neq E$ be a primary element of $\operatorname{Cs} A_{2}$. If $X$ belongs to $\mathfrak{u}$, then $\boldsymbol{C s} X=\boldsymbol{C} \boldsymbol{s} A_{2}$. Let $X$ belong to $\mathfrak{P}_{2}$ and let $\boldsymbol{C} \boldsymbol{s} X$ be not contained in $\boldsymbol{C s} A_{2}$. By a theorem of Wielandt [5, p. 285] $\boldsymbol{C s} X$ is nilpotent. Hence $\boldsymbol{C s} X \subseteq \boldsymbol{C} \boldsymbol{u} \mathfrak{u}=\boldsymbol{C} \boldsymbol{s} A_{2}$. This is a contradiction. Hence $\boldsymbol{C s} A_{2}$ is centralizer-closed. This contradicts [9].

Let $B_{5}$ be an element of (S) such that $\boldsymbol{C} \boldsymbol{s} A_{2} \supseteqq \boldsymbol{C} \boldsymbol{s} B_{5}$ and such that (3) : $\boldsymbol{C s} B_{5}=n_{5}$. Then by a theorem of Camina [2] $n_{5} / n_{2}$ is a power of $p_{2}$ and $Z\left(\boldsymbol{C s} A_{2}\right)$ is a $p_{2}$-group.

(2.7) The Sylow $p_{2}$-complement $\mathfrak{u}$ of $\boldsymbol{C s} B_{5}$ and moreover $\boldsymbol{C s} B_{5}$ itself are abelian.

Proof. First we show that $\mathfrak{U}$ is abelian. If $\pi(\mathfrak{U})$ contains at least two prime numbers, this is obvious. So let us assume that $\mathfrak{U}$ is a $q$-group, where $q$ is a prime. Let $B \neq E$ be an element of $\mathfrak{u}$. Then $\boldsymbol{C s} B \subseteq \boldsymbol{C} \boldsymbol{s} A_{2}$. In fact, otherwise, $|\boldsymbol{C} \boldsymbol{s} B|=\left|\boldsymbol{C} \boldsymbol{s} A_{2}\right|$. Then by a theorem of Camina [2] CsB is nilpotent. Then by a theorem of Wielandt [5, p. 285] $\boldsymbol{C} \boldsymbol{s} A_{2}$ is nilpotent against (2.6). Hence $\boldsymbol{C s} B$ is a conjugate of $\mathfrak{u}$ in $\boldsymbol{C s} A_{2}$. By a theorem of Burnside [5, p. 492] Cs $A_{2}$ is solvable. Thus a theorem of Fitting [5, p. 277] implies 
that $\mathfrak{U}$ is abelian. The rest is obvious.

(2.8) $\left|C s A_{2}\right|$ is odd.

Proof. Assume that $\left|\boldsymbol{C s} A_{2}\right|$ is even. By a theorem of Walter [13] and by (2.7) $p_{2}=2$. By the proofs of (4.5) and (4.6) in [8] there exists a 2-element $B$ such that $|\boldsymbol{C s} B|<\left|\boldsymbol{C} \boldsymbol{s} A_{2}\right|$. By the proof of (2.7) $C s B$ is abelian. Therefore we may assume that $B=B_{5}$ and that $\boldsymbol{C s} B$ is contained in $\boldsymbol{C s} A_{2}$.

Since $\boldsymbol{C s} B_{5}$ is nilpotent and since $\boldsymbol{C s} A_{2}=\mathfrak{P}_{2} \boldsymbol{C s} B_{5}, \boldsymbol{C s} A_{2}$ is solvable [5, p. 674]. Let $\mathfrak{P}_{2}^{*}$ be the Sylow 2-subgroup of $\boldsymbol{C s} B_{5}$. If $\boldsymbol{F}\left(\boldsymbol{C s} A_{2}\right)$ is a 2-group, then by a theorem of Fitting [5, p. 277] $\boldsymbol{F}\left(\boldsymbol{C s} A_{2}\right) \neq \mathfrak{P}_{2}^{*}$. Now $\left(\boldsymbol{F}\left(\boldsymbol{C s} A_{2}\right) \cap \boldsymbol{N} s \mathfrak{P}_{2}^{*}\right) / \mathfrak{P}_{2}^{*}$ is the kernel of a Frobenius group $\left(\boldsymbol{F}\left(\boldsymbol{C s} A_{2}\right) \cap \boldsymbol{N} \mathfrak{s}_{2}^{*}\right) \mathfrak{u} / \mathfrak{P}_{2}^{*}$. Let $A$ be an element of $\boldsymbol{F}\left(\boldsymbol{C s} A_{2}\right) \cap \boldsymbol{N} \boldsymbol{s} \mathfrak{P}_{2}^{*}$ outside $\mathfrak{P}_{2}^{*}$. If $A^{-1} \mathfrak{U} A \neq \mathfrak{U}$, then $\boldsymbol{C s} B_{5}$ contains $A^{-1} \mathfrak{U} A$. This is a contradiction. If $A^{-1} \mathfrak{U} A=\mathfrak{u}$, then $[A, \mathfrak{u}]$ is contained in $\mathfrak{u} \cap \boldsymbol{F}\left(\boldsymbol{C s} A_{2}\right)$ $=$ (5. This is a contradiction. Hence $\boldsymbol{F}\left(\boldsymbol{C s} A_{2}\right)=\boldsymbol{C s} B_{5}$. Then $\boldsymbol{C s} A_{2} / \mathfrak{P}_{2}^{*}$ is a Frobenius group with $\boldsymbol{C s} B_{5} / \mathfrak{P}_{2}^{*}$ the kernel. Hence $\mathfrak{P}_{2} / \mathfrak{P}_{2}^{*}$ is cyclic or generalized quaternion.

First assume that $\mathfrak{P}_{2} / \mathfrak{P}_{2}^{*}$ is a generalized quaternion group of order $2^{a}$. Then there exist elements $Q$ and $R$ of $\mathfrak{P}_{2}$ and $S, T, U$ and $V$ of $\mathfrak{P}_{2}^{*}$ such that $R^{-1} Q R=Q^{-1} S, Q^{2^{a-2}}=R^{2} T, Q^{2^{a-1}}=U, R^{4}=V$ and $\mathfrak{P}_{2} / \mathfrak{P}_{2}^{*}=\langle Q, R\rangle \mathfrak{P}_{2}^{*} / \mathfrak{P}_{2}^{*}$. Now suppose that $\mathfrak{P}_{2}^{*}$ is not cyclic. Let $\mathfrak{W}$ be a normal subgroup of type (2.2) of $\mathfrak{P}_{2}$ contained in $\mathfrak{P}_{2}^{*}$. Then $\boldsymbol{C s} R^{2}$ contains $\mathfrak{W}$. If $\left|\boldsymbol{C s} R^{2}\right|=\left|\boldsymbol{C s} B_{5}\right|$, then by (2.7) $\boldsymbol{C s} R^{2}$ is abelian. This implies that $C s R^{2} \subseteq C s A_{2}$ and that $R^{2}$ belongs to $\mathfrak{F}_{2}^{*}$. This is a contradiction. Hence $\left|\boldsymbol{C s} R^{2}\right|=\left|\boldsymbol{C s} A_{2}\right|$. If $\boldsymbol{F}\left(\boldsymbol{C s} R^{2}\right)$ is a 2 -group, then we have that $\mathfrak{P}_{2}: \mathfrak{F}_{2}^{*}>|\mathfrak{U}|$. This is a contradiction. Hence $\boldsymbol{F}\left(\boldsymbol{C s} R^{2}\right)$ is not a 2-group. Let $\overline{\mathfrak{F}}_{2}$ and $\overline{\mathfrak{u}}$ be the Sylow 2complement of $\boldsymbol{F}\left(\boldsymbol{C s} R^{2}\right)$, respectively. Let $\widehat{\mathfrak{F}}_{2}$ be a Sylow 2-subgroup of $\boldsymbol{C s} R^{2}$. Then $\widehat{\mathfrak{P}}_{2} / \overline{\mathfrak{F}}_{2}$ is cyclic or generalized quaternion. This implies that $\mathfrak{W} \cap \overline{\mathfrak{F}}_{2} \neq \mathfrak{F}$. Take an element $W(\neq E)$ of $\mathfrak{W} \cap \overline{\mathfrak{P}}_{2}$. Then $\boldsymbol{C s} W$ contains $\overline{\mathfrak{U}}$ and $\overline{\mathfrak{U}}$. This implies that $\mathfrak{U}=\overline{\mathfrak{U}}$. This is a contradiction. Therefore $\mathfrak{P}_{2}^{*}$ is cyclic. Hence $\mathfrak{P}_{2} \cap \boldsymbol{C s} \mathfrak{F}_{2}^{*} \neq \mathfrak{P}_{2}^{*}$. Thus $\boldsymbol{C s} Q^{2^{a-2}}$ 
contains $\mathfrak{P}_{2}^{*}$. This implies that $\left|\boldsymbol{C s} Q^{2^{a-2}}\right|=\left|\boldsymbol{C s} A_{2}\right|$. If $\boldsymbol{F}\left(\boldsymbol{C s} Q^{2^{a-2}}\right)$ is a 2-group, then we have that $\mathfrak{F}_{2}: \mathfrak{B}_{2}^{*}>|\mathfrak{U}|$. This contradiction shows that $\boldsymbol{F}\left(\boldsymbol{C s} Q^{2^{a-2}}\right)$ is not a 2-group. If $\boldsymbol{C s} Q \neq \boldsymbol{C s} Q^{2^{a-2}}$, then $\boldsymbol{C s} Q=\boldsymbol{F}$ $\left(\boldsymbol{C} s Q^{2 a-2}\right)$. Let $\mathfrak{P}_{2}^{\sharp}$ be the Sylow 2-subgroup of $\boldsymbol{C s} Q$. Then $\left[Q, \mathfrak{P}_{2}^{*}\right]$ $\subseteq \mathfrak{P}_{2}^{*} \cap \mathfrak{P}_{2}^{*}=\mathfrak{F}$. Since $|\boldsymbol{C s} Q|=\left|\boldsymbol{C s} B_{5}\right|$, this is a contradiction. Hence $\boldsymbol{C s} Q=\boldsymbol{C s} Q^{2^{a-2}}$. Similarly we obtain that $\boldsymbol{C s} R=\boldsymbol{C s} R^{2}$. Since $Q^{2^{a-2}}$ and $R^{2}$ commute, this implies that $Q$ and $R$ commute. This is a contradiction. Therefore $\mathfrak{P}_{2} / \mathfrak{P}_{2}^{*}$ is cyclic.

Let $\mathfrak{P}_{2} / \mathfrak{P}_{2}^{*}$ be of order $2^{a}$ and $P \mathfrak{P}_{2}^{*}$ a generator of $\mathfrak{F}_{2} / \mathfrak{F}_{2}^{*}$. Assume that $a \geqq 2$. As above, we obtain that $\mathfrak{P}_{2}^{*}$ is cyclic. Therefore, $\mathfrak{P}_{2}$ is metacyclic. Then by a theorem of Mazurov [10] $\mathfrak{P}_{2}$ is of type (2.2) or of maximal class. This is a contradiction. Hence we obtain that $a=1$. Now we show that $\boldsymbol{Z}\left(\mathfrak{P}_{2}\right)$ is of order 2 . Assume the contrary. If $|\boldsymbol{C s} P|=\left|\boldsymbol{C s} B_{5}\right|$, then by (2.7) $\boldsymbol{C s} P$ is abelian and $\boldsymbol{C s} P \cap \mathbb{U}=(\tilde{\xi}$. Let $\mathfrak{u}^{\sharp}$ be the Sylow 2-complement of $\boldsymbol{C s} P$. Then $\mathfrak{u} \cap \mathfrak{u}^{\sharp}=\mathfrak{r}$. But since $\boldsymbol{C} \boldsymbol{s} P$ contains $\boldsymbol{Z}\left(\mathfrak{P}_{2}\right)$, this is a contradiction. If $|\boldsymbol{C s} P|=\left|\boldsymbol{C s} A_{2}\right|$, then let $\widehat{\mathfrak{P}}_{2}$ and $\widehat{\mathfrak{u}}$ be the Sylow 2-subgroup and Sylow 2-complement of $\boldsymbol{F}(\boldsymbol{C} \boldsymbol{s} P)$. Then $\mathfrak{P}_{2}^{*} \cap \widehat{\aleph}_{2} \neq(\mathfrak{s}$ by assumption. Let $Z(\neq E)$ be an element of $\mathfrak{P}_{2}^{*} \cap \widehat{\mathfrak{F}}_{2}$. $\quad \boldsymbol{C s} Z$ contains $\mathfrak{u}$ and $\widehat{\mathfrak{u}}$. Since $\mathfrak{u} \cap \widehat{\mathfrak{u}}=\mathfrak{F}$, and since $\boldsymbol{F}(\boldsymbol{C} \boldsymbol{s} Z)$ contains $\mathfrak{U}$ and $\hat{\mathfrak{u}}$, this is a contradiction. Hence $\left|\boldsymbol{Z}\left(\mathfrak{P}_{2}\right)\right|=2$. Then by a lemma of Suzuki [11] $\mathfrak{P}_{2}$ is of type $(2,2)$ or of maximal class. Then by a theorem of Wong [14] we get a contradiction.

(2. 9) $\quad \boldsymbol{F}\left(\boldsymbol{C s} A_{2}\right)$ is a $p_{2}$-group.

Proof. Assume the contrary. Then $\boldsymbol{F}\left(\boldsymbol{C s} A_{2}\right)=\boldsymbol{C s} B_{5}=\mathfrak{B}_{2}^{*} \times \mathfrak{U}$. Since $\boldsymbol{F}\left(\boldsymbol{C} \boldsymbol{s} A_{2}\right) / \mathfrak{P}_{2}^{*}$ is the kernel of a Frobenius group $\boldsymbol{C} \boldsymbol{s} A_{2} / \mathfrak{P}_{2}^{*}$, $\mathfrak{P}_{2} / \mathfrak{P}_{2}^{*}$ is cyclic by (2.8). Let $\mathfrak{P}_{2} / \mathfrak{P}_{2}^{*}$ be of order $p_{2}^{a}$ and $P \mathfrak{P}_{2}^{*}$ a generator of $\mathfrak{F}_{2} / \mathfrak{F}_{2}^{*}$. Assume that $a \geqq 2$. Then as in the proof of (2.8) we obtain that $\mathfrak{F}_{2}^{*}$ is cyclic. Therefore $\mathfrak{P}_{2}$ is metacyclic. If $\mathfrak{P}$, is not abelian, then by a theorem of Huppert [5, p. 452] (S) is not simple. Hence $\mathfrak{P}_{2}$ is abelian. Since $\langle P\rangle \cap \mathfrak{P}_{2}^{*}=\mathfrak{F}$, we obtain that $\mathfrak{\beta}_{2}=\mathfrak{\beta}_{2}^{*} \times\langle P\rangle$ is of type $\left(p_{2}^{a}, p_{2}^{a}\right)$. 
Now the set of elements $X$ of (S) such that (S): $\boldsymbol{C} \boldsymbol{s} X=n_{2}$ coincides with the set of $p_{2}$-elements $\neq E$ in (S). Every $p_{2}$-element $\neq E$ belongs to exactly one conjugate of $\mathfrak{P}_{2}^{*}$. Now $\mathbf{N} \mathfrak{P}_{2}^{*}=\boldsymbol{C} \boldsymbol{s} A_{2}$. In fact, otherwise, since $\boldsymbol{C} \boldsymbol{s}_{2}^{*}=\boldsymbol{C} \boldsymbol{s} A_{2}$, by a theorem of Thompson [5, p. 499] we obtain that $\boldsymbol{C s} A_{2}$ is nilpotent contradicting (2.6). Let e be the number of conjugacy classes of elements $X$ of \&S such that (S) : Cs $X$ $=n_{2}$. Then we obtain that

$$
e n_{2}=n_{2}\left(p_{2}^{a}-1\right) \text {. }
$$

Hence $e=p_{2}^{a}-1$. On the other hand, by a theorem of Burnside [5, p. 418] any two elements of $\mathfrak{P}_{2}$ which are conjugate in $(5)$ are conjugate in $\boldsymbol{N} \boldsymbol{s} \mathfrak{F}_{2}$. Since $\boldsymbol{C} \boldsymbol{s} \mathfrak{P}_{2}=\mathfrak{P}_{2}$, we obtain that $\boldsymbol{N} \boldsymbol{s} \mathfrak{P}_{2}: \mathfrak{F}_{2}=p_{2}^{a}+1$. In particular, there exists an involution $J$ in $\mathbf{N} \mathfrak{Y}_{2}$ such that $J$ inverts $A_{2}$. Then by a theorem of Thompson [5, p. 499] we obtain that $\boldsymbol{C s} A_{2}$ is nilpotent contradicting (2.6) Hence we obtain that $a=1$.

If $|\boldsymbol{C} \boldsymbol{s} P|=\left|\boldsymbol{C} \boldsymbol{s} B_{5}\right|$, then by (2.7) $\boldsymbol{C s} P$ is abelian. Then $\boldsymbol{C s} P$ is contained in $\boldsymbol{C s} A_{2}$. This is a contradiction. Hence $|\boldsymbol{C s} P|=\left|\boldsymbol{C s} A_{2}\right|$. Let $\widehat{\mathfrak{F}}_{2}$ be the Sylow $p_{2}$-subgroup of $\boldsymbol{F}(\boldsymbol{C s} P)$. Since $\widehat{\mathfrak{F}}_{2} \cap \mathfrak{P}_{2}^{*}=\mathfrak{F}$, we have that $\left|\mathfrak{F}_{2}^{*} \cap \boldsymbol{C s} P\right|=p_{2}$. If $\mathfrak{P}_{2}$ is abelian, we get a contradiction as above. So we may assume that $\mathfrak{P}_{2}$ is not abelian. Hence we have that $\left|\mathfrak{P}_{2}\right|=p_{2}^{3}$. By the transfer theorem of Wielandt $[5, \mathrm{p} .447]$ $\boldsymbol{N} \mathfrak{P}_{2} \neq \mathfrak{F}_{2}$. Since $\boldsymbol{Z}\left(\mathfrak{P}_{2}\right)=\left\langle A_{2}\right\rangle$ we have that $\boldsymbol{C s} A_{2} \neq \boldsymbol{N} \boldsymbol{s}\left\langle A_{2}\right\rangle$. Then by a theorem of Thompson [5, p. 499] $\boldsymbol{C} \boldsymbol{s} A_{2}$ is nilpotent against (2.6).

Remark. The proof of (2.10) of [8] is incomplete, because it leaves open the case where $\mathfrak{P}_{2}$ is abelian but not cyclic. The proof of (2.10) of [8] can be completed as above. But meanwhile Camina [2 [ has found an essentially simpler proof to kill the 2-headed case for the conjugate type rank 4 simple groups.

(2.10) Let $X \neq E$ be a $p_{2}$-element of $\&$. Then $|\boldsymbol{C s} X|=\left|\boldsymbol{C s} A_{2}\right|$.

Proof. Assume that $|\boldsymbol{C s} X| \neq\left|\boldsymbol{C s} A_{2}\right|$. By (2.7) $\boldsymbol{C s} X$ is abelian. 
Hence we may assume that $\boldsymbol{C} \boldsymbol{s} X \subseteq \boldsymbol{C} \boldsymbol{s} A_{2}$. Let $\widehat{\mathfrak{H}}_{2}$ and $\widehat{\mathfrak{U}}$ be the Sylow $p_{2}$-subgroup and Sylow $p_{2}$-complement of $\boldsymbol{C s} X$, respectively. By (2.9) $\boldsymbol{F}\left(\boldsymbol{C} s A_{2}\right) \neq \widehat{\mathfrak{F}}_{2}$. Hence $\boldsymbol{F}\left(\boldsymbol{C} \boldsymbol{s} A_{2}\right) \cap \boldsymbol{N} \boldsymbol{s} \widehat{\mathfrak{⿰}}_{2} \neq \widehat{\mathfrak{F}}_{2}$. Let $X_{1}$ be an element of $\boldsymbol{F}\left(\boldsymbol{C} \boldsymbol{s} A_{2}\right) \cap \boldsymbol{N} \widehat{\mathfrak{⿰}}_{2}$ outside $\widehat{\mathfrak{W}}_{2}$. Then $\left[\widehat{\mathfrak{F}}_{2}, X_{1}^{-1} \widehat{\mathfrak{u}} X_{1}\right]=\mathfrak{F}$. If $X_{1}^{-1} \widehat{\mathfrak{u}} X_{1}=\widehat{\mathfrak{u}}$, then $\left[X_{1}, \widehat{\mathfrak{u}}\right]=\boldsymbol{F}\left(\boldsymbol{C s} A_{2}\right) \cap \widehat{\mathfrak{u}}=\mathbb{F}$. This is a contradiction.

(2.11) $\mathfrak{P}_{2}$ is of exponent $p_{2}$.

Proof. Assume that $\mathfrak{F}_{2}$ is of exponent $p_{2}^{a}$, where $a \geqq 2$. Then by (2.10) we may assume that $\boldsymbol{Z}\left(\boldsymbol{C s} A_{2}\right)$ contains an element $C$ of order $p_{2}^{2}$. Let $X$ be an element of $\boldsymbol{C s} A_{2}$ of order $p_{2}$. Then $\boldsymbol{C s} C X=$ $\boldsymbol{C s} C^{p_{2}}=\boldsymbol{C s} A_{2}$. Hence all elements of $\boldsymbol{C s} A_{2}$ of order $p_{2}$ belong to $\boldsymbol{Z}$ $\left(\boldsymbol{C} \boldsymbol{s} A_{2}\right)$. This implies that $\mathfrak{P}_{2}=\boldsymbol{Z}\left(\boldsymbol{C s} A_{2}\right)$. Then by (2.9) $\boldsymbol{F}\left(\boldsymbol{C} \boldsymbol{s} A_{2}\right)=\mathfrak{P}_{2}$. Hence $\boldsymbol{C s} A_{2} \cap \boldsymbol{N} \boldsymbol{s} \mathfrak{U}=\boldsymbol{C s} \mathfrak{u}$. If $\boldsymbol{N} \mathfrak{s} \mathfrak{U}=\boldsymbol{C s} \mathfrak{u}$, then by the transfer theorem of Burnside (S) is not simple. Hence $N \boldsymbol{s} \mathfrak{u} \neq \boldsymbol{C} \boldsymbol{s} \mathfrak{u}$. Let $V$ be an element of $\boldsymbol{N} \mathfrak{s} \mathfrak{U}$ outside $\boldsymbol{C s} \mathfrak{u}$. Since $\boldsymbol{C s} \mathfrak{U}=\mathfrak{P}_{2}^{*} \times \mathfrak{U}, V$ normalizes $\mathfrak{P}_{2}^{*}$. Since $\boldsymbol{C s} \mathfrak{P}_{2}^{*}=\boldsymbol{C s} A_{2}, V$ belongs to $\boldsymbol{N s}\left(\boldsymbol{C s} A_{2}\right)$, but not to $\boldsymbol{C s} A_{2}$. Hence by a theorem of Thompson [5, p. 499] $\boldsymbol{C} s A_{2}$ is nilpotent. This is a contradiction.

(2.12) $\pi\left(C s A_{2}\right)=\pi(N s \mathfrak{U})$.

Proof. If $s$ is a prime of $\pi(\boldsymbol{C s} \mathfrak{u})$ not belonging to $\pi\left(\boldsymbol{C s} A_{2}\right)$, then let $S \neq E$ be an s-element of $N \boldsymbol{s} \mathfrak{u}$. Then $\mathrm{S}$ normalizes $\mathfrak{P}_{2}^{*}$ and hence $\boldsymbol{C} \boldsymbol{s}_{2}^{*} . \quad\langle S\rangle \boldsymbol{C s} \mathfrak{F}_{2}^{*}$ is a Frobenius group with $\boldsymbol{C s} \mathfrak{F}_{2}^{*}$ the kernel. By a theorem of Thompson [5, p. 499], $\boldsymbol{C} \boldsymbol{s}_{2}^{*}$ is nilpotent. By the proof of (2.10) $\boldsymbol{C} \boldsymbol{s} \Re_{2}^{*}$ contains $\boldsymbol{C s} \mathfrak{u}$ properly. This is a contradiction. If $p_{2}$ does not belong to $\pi(N \boldsymbol{s} \mathfrak{u})$ then by the transfer theorem of Burnside (S) is not simple.

Now we get a desired contradiction as follows.

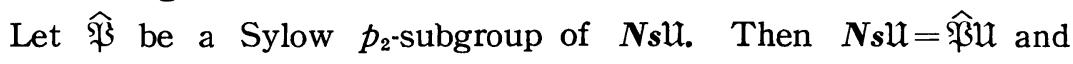
$\widehat{\mathfrak{P}} \neq \mathfrak{F}$ by (2.12). Notice that $\boldsymbol{C s} \mathfrak{U}=\mathfrak{P}_{2}^{*} \times \mathfrak{U}$, where $\mathfrak{H}_{2}^{*}$ contains $A_{\mathbf{2}}$. Thus $\mathfrak{P}_{2}^{*} \cap Z(\widehat{\mathfrak{P}}) \neq\left(\mathfrak{F}\right.$. Let $A^{\prime} \neq E$ be an element of $\mathfrak{\beta}_{2}^{*} \cap \boldsymbol{Z}(\widehat{\mathfrak{P}})$. Then $\boldsymbol{C s} A^{\prime}$ contains $N \boldsymbol{s} \mathfrak{u}$. Let $\overline{\mathfrak{P}}$ be a Sylow $p_{2}$-subgroup of $\boldsymbol{C} s A^{\prime}$. Since 
$\boldsymbol{N} \mathfrak{u} \neq \boldsymbol{C} \boldsymbol{s} \mathfrak{u}, \boldsymbol{F}\left(\boldsymbol{C s} A^{\prime}\right) \neq \overline{\mathfrak{P}} . \quad \boldsymbol{N} \boldsymbol{s} \mathfrak{U} / \mathfrak{P}_{2}^{*}$ is a Frobenius group with $\boldsymbol{C s} \mathfrak{u} / \mathfrak{P}_{2}^{*}$ the kernel. Since $\boldsymbol{F}\left(\boldsymbol{C} \boldsymbol{s} A^{\prime}\right) \cap \boldsymbol{N} \boldsymbol{s} \mathfrak{U}=\boldsymbol{F}\left(\boldsymbol{C} \boldsymbol{s} A^{\prime}\right) \cap \boldsymbol{C} \boldsymbol{s} \mathfrak{U}$ and since $\overline{\mathfrak{P}}=\boldsymbol{F}$ $\left(\boldsymbol{C} \boldsymbol{s} A^{\prime}\right)(\overline{\mathfrak{P}} \cap \boldsymbol{N} \boldsymbol{u} \mathfrak{U}), \overline{\mathfrak{S}} \cap \mathbf{N s} \mathfrak{U} / \mathfrak{P}_{2}^{*}$ is cyclic. Hence $\overline{\mathfrak{P}}: \boldsymbol{F}\left(\boldsymbol{C} \boldsymbol{s} A^{\prime}\right)=p_{2}$. Put $\boldsymbol{N} \boldsymbol{s} \overline{\mathfrak{B}} \cap \boldsymbol{C} \boldsymbol{s} A^{\prime}=\overline{\mathfrak{B}} \overline{\mathfrak{U}}$, where $\overline{\mathfrak{u}}$ is a subgroup of $\mathfrak{u}$. If $\overline{\mathfrak{U}} \neq \mathfrak{r}$, then let $X \neq E$ be an element of $N s \mathfrak{u} \cap \overline{\mathfrak{Y}}$ outside $\mathfrak{P}_{2}^{*}$. Then $[X, \overline{\mathfrak{u}}]=\overline{\mathfrak{P}} \cap \overline{\mathfrak{u}}=\mathfrak{r}$. Since $\boldsymbol{C} \boldsymbol{s} \overline{\mathfrak{U}}=\boldsymbol{C} \boldsymbol{s} \mathfrak{U}=\mathfrak{F}_{2}^{*} \times \mathfrak{U}$, this is a contradiction. Hence $\boldsymbol{N} \overline{\mathfrak{P}} \cap \boldsymbol{C s} A^{\prime}$ $=\overline{\mathfrak{S}}$. By the transfer theorem of Wielandt $\left[5\right.$, p. 447] $\boldsymbol{C} s A^{\prime}$ is $p_{2^{-}}$ nilpotent. This is a contradiction.

\section{4-headed case}

Let $(3)$ be a simple group of conjugate type rank 5 and of 4 headed. Let $n_{i}$ be maximal elements of $I(\&)(i=1,2,3,4)$. Let $A_{i}$ be an element of \&S such that (S) : $\boldsymbol{C} \boldsymbol{s} A_{i}=n_{i}(i=1,2,3,4)$.

Part $A$. The purpose of this part is to prove that at least one of the $\boldsymbol{C s} A_{i}(i=1,2,3,4)$ is free.

Assume the contrary. Then let $X_{i}$ be an element of (S) such that $\boldsymbol{C s} X_{i}$ is properly contained in $\boldsymbol{C s} A_{i}(i=1,2,3,4)$. Thus (S) : $\boldsymbol{C s} X_{i}=n_{5}(i=1,2,3,4)$.

(3A. 1) $\boldsymbol{C s} A_{i}$ is not nilpotent $(i=1,2,3,4)$.

Proof. Assume that $\boldsymbol{C s} A_{1}$ is nilpotent. Obviously there exists a nonabelian Sylow $p_{1}$-subgroup $\mathfrak{P}_{1}$ of $\boldsymbol{C s} A_{1}$, where $p_{1}$ is a prime. We may assume that $A_{1}$ is an element of $\boldsymbol{Z}\left(\mathfrak{P}_{1}\right)$. Hence $\mathfrak{P}_{1}$ is a Sylow $p_{1}$-subgroup of $\mathbb{S}$. Let $\mathfrak{U}$ be the Sylow $p_{1}$-complement of $\boldsymbol{C} s A_{1}$. Clearly $\mathfrak{U}$ is abelian. Since $\boldsymbol{C s} A_{1}$ is not a Hall subgroup of $\mathbb{F}$, there exists a prime $q$ in $\pi(\mathfrak{U})$ such that the Sylow $q$-subgroup $\mathfrak{Q}$ of $\boldsymbol{C s} A_{1}$ is not a Sylow $q$-subgroup of $(S)$. Then there exists a $q$-element $Q \neq E$ of $\mathfrak{Q}$ such that a Sylow $q$-subgroup of $C s Q$ contains $\mathfrak{Q}$ properly. Since $\boldsymbol{C s} A_{1}$ is contained in $\boldsymbol{C s} Q$, this is a contradiction.

Now by a theorem of Camina [2] we obtain that $\operatorname{Cs} A_{i}: \operatorname{Cs} X_{i}=$ $p_{i}^{a_{i}}$, where $p_{i}$ is a prime, and that $\boldsymbol{Z}\left(\boldsymbol{C s} A_{i}\right)$ is a $p_{i}$-group $(i=1,2,3$, 4). By the choice of $A_{i}$ the $p_{i}$ are distinct. 
(3A.2) $\pi(\mathbb{S})=\left\{p_{1}, p_{2}, p_{3}, p_{4}\right\}$

Proof. Let $q$ be a prime divisor of $|\mathbb{S}|$ distinct from $p_{i}$ ( $i=$ $1.2,3,4)$. We may assume that $\boldsymbol{C} \boldsymbol{s} A_{1}$ contains a Sylow $q$-subgroup $\mathfrak{Q}$ of $\mathbb{G}$. Let $Q \neq E$ be an element of $\boldsymbol{Z}(\mathfrak{Q})$. Then we have that $\boldsymbol{C s} A_{1} Q$ contains $\mathfrak{Q}$ and that $\left|\boldsymbol{C s} A_{1} Q\right|=\left|\boldsymbol{C s} X_{1}\right|$. This shows that $\boldsymbol{C} \Omega \mathfrak{\text { and }}$ its conjugates exhaust (\$). Hence $\mathbb{G}=\boldsymbol{C} \boldsymbol{s} \cong$. This contradicts the simplicity of 18 .

(3A. 3) Let $|\boldsymbol{C s} X|=\left|\boldsymbol{C s} X_{1}\right|$. Then $\boldsymbol{C s} X$ is abelian.

Proof. This is obvious, since $p_{1} p_{2} p_{3} p_{4}$ divides $|\boldsymbol{C s} X|$ and since $\boldsymbol{C s} A_{i}: \boldsymbol{C s} X_{i}$ is a power of $p_{i}(i=1,2,3,4)$

(3A. 4) We may choose $X=X_{1}$ and $A_{i}(i=1,2,3,4)$ so that Cs $X$ is contained in $\bigcap_{i=1}^{4} \boldsymbol{C s} A_{i}$.

Proof. We show that $\boldsymbol{C s} X$ contains a $p_{i}$-element $A_{i}^{\prime}(i>1)$ such that $\boldsymbol{C} \boldsymbol{s} X$ is contained in $\boldsymbol{C} \boldsymbol{s} A_{i}^{\prime}$ and that $\left|\boldsymbol{C} \boldsymbol{s} A_{i}^{\prime}\right|=\left|\boldsymbol{C} \boldsymbol{s} A_{i}\right|$. Let $A_{i}^{\prime \prime} \neq E$ be any $p_{i}$-element of $\boldsymbol{C s} X$. We may assume that $A_{i}^{\prime \prime}$ belongs to $\boldsymbol{C s} A_{i}$. If $\boldsymbol{C} \boldsymbol{s} A_{i}^{\prime \prime}=\boldsymbol{C} \boldsymbol{s} X$, then $\boldsymbol{C} \boldsymbol{s} X$ contains $A_{i}$. Put $A_{i}^{\prime}=A_{i}$. If $\left|\boldsymbol{C} \boldsymbol{s} A_{i}^{\prime \prime}\right|=\left|\boldsymbol{C} \boldsymbol{s} A_{i}\right|$, put $A_{i}^{\prime}=A_{i}^{\prime \prime}$.

Let $\mathfrak{B}_{i}$ be a Sylow $p_{i}$-subgloup of $\boldsymbol{C s} A_{i}$. Then by (3A. 4) $\boldsymbol{C s} A_{i}$ $=\mathfrak{B}_{i} \boldsymbol{C s} X$. In particular, $\boldsymbol{C s} A_{i}$ is solvable $(i=1,2,3,4)$ [5, p. 674].

(3A.5) For at least one $i, \boldsymbol{F}\left(\boldsymbol{C s} A_{i}\right)$ is a $p_{i}$-group.

Proof. Assume the contrary. Then $\boldsymbol{C s} X=\boldsymbol{F}\left(\boldsymbol{C} \boldsymbol{s} A_{i}\right) \quad(i=1,2,3$, 4). Hence $\mathbb{B}=\boldsymbol{N} \boldsymbol{s}(\boldsymbol{C s} X)$. This contradicts the simplicity of $\mathbb{B}$.

We assume that $\boldsymbol{F}\left(\boldsymbol{C} \boldsymbol{s} A_{1}\right)$ is a $p_{1}$-group. Let $\mathfrak{P}_{i}^{*}$ be the Sylow $p_{i}$-subgroup of $\boldsymbol{C s} X(i=1,2,3,4)$.

(3A. 6) For at least three $i$ 's, $\boldsymbol{F}\left(\boldsymbol{C s} A_{i}\right)$ is a $p_{i}$-group.

Proof. By a theorem of Fitting [5, p. 277] we have that $\boldsymbol{F}\left(\boldsymbol{C} \boldsymbol{s} A_{1}\right)$ contains $\mathfrak{P}_{1}^{*}$ properly. Then $\left(\boldsymbol{F}\left(\boldsymbol{C} \boldsymbol{s} A_{1}\right) \cap \boldsymbol{N s} \mathfrak{S}_{1}^{*}\right) \mathfrak{B}_{2}^{*} \mathfrak{\mathfrak { F } _ { 3 } ^ { * }} \mathfrak{B}_{4}^{*} / \mathfrak{B}_{1}^{*}$ 
is a Frobenius group with $\boldsymbol{F}\left(\boldsymbol{C s} A_{1}\right) \cap \boldsymbol{N} \boldsymbol{s} \Re_{1}^{*} / \mathfrak{P}^{k}$ the kernel. Therefore $\mathfrak{P}_{2}^{*} \mathfrak{P}_{3}^{*} \mathfrak{P}_{4}^{*}$ is cyclic. Now assume that $\boldsymbol{F}\left(\boldsymbol{C} s A_{i}\right)$ is not a $p_{i}$-group for $i=3$, 4. Then $\boldsymbol{F}\left(\boldsymbol{C} \boldsymbol{s} A_{i}\right)=\boldsymbol{C} \boldsymbol{s} X$ for $i=3,4$. We may assume that $p_{3}>p_{4}$. Since $\mathfrak{P}_{4}^{*}$ is cyclic, we may assume that $\mathfrak{P}_{3} \mathfrak{P}_{4}^{*}$ is $p_{4}$-nilpotent. Hence $\left[\mathfrak{P}_{3}, \mathfrak{P}_{4}^{*}\right] \subseteq \mathfrak{P}_{3} \cap \mathfrak{P}_{4}^{*}=(\mathfrak{F}$. This is a contradiction.

We assume that $\boldsymbol{F}\left(\boldsymbol{C} \boldsymbol{s} A_{i}\right)$ is a $p_{i}$-group for $i=1,2,3$.

(3A. 7) If $\boldsymbol{F}\left(\boldsymbol{C s} A_{4}\right)$ is not a $p_{4}$-group, then $p_{4}<p_{i}(i=1,2,3)$.

Proof. If so, we have that $\boldsymbol{F}\left(\boldsymbol{C s} A_{4}\right)=\boldsymbol{C s} X$. By the proof of (3A. 6) Cs $X$ is cyclic. Since $\mathfrak{P}_{i}^{*} \mathfrak{P}_{4} / \mathfrak{P}_{4}^{*}$ is a Frobenius group with $\mathfrak{P}_{i}^{*} \mathfrak{P}_{4}^{*} / \mathfrak{P}_{*}^{*}$ the kernel, $p_{i}>p_{4}(i=1,2,3)$

Now we may assume that $p_{1}>p_{2}>p_{3}>p_{4}$. Then $\boldsymbol{F}\left(\boldsymbol{C s} A_{1}\right)=\mathfrak{P}_{1}$.

We show that $N \boldsymbol{s}_{\mathfrak{P}_{1}}$ and its conjugates exhaust (S). Let $G \neq E$ be any element of (S). If $|\boldsymbol{C s} G|=\left|\boldsymbol{C s} A_{1}\right|$, then (S) is a $p_{1}$-element. If $|\boldsymbol{C s} G|=\left|\boldsymbol{C s} A_{i}\right|$ for $i>1$, then $G$ is a $p_{i}$-element. Since $\boldsymbol{C s} G$ is not free, there exists an element $H$ in $\boldsymbol{C s} G$ such that $\boldsymbol{C s} H$ is properly contained in $\boldsymbol{C s} G$. $G$ belongs to $\boldsymbol{C s H}$. By the proof of (3A.4) there exists a $p_{1}$-element $A_{1}^{\prime} \neq E$ such that $\boldsymbol{C s} H$ is contained in $\boldsymbol{C s} A_{1}^{\prime}$ and that $\left|\boldsymbol{C s} A_{1}^{\prime}\right|=\left|\boldsymbol{C s} A_{1}\right|$. Therefore, $\boldsymbol{N} \boldsymbol{s} \mathfrak{P}_{1}=$ (S) and (5) is not simple. This is a cntradiction.

Part $B$. We use the same notation as in Part $A$. By Part $A$. we may assume that $\boldsymbol{C s} A_{4}$ is free. The purpose of this part is to prove that at least one of $\boldsymbol{C s} A_{i}(i=1,2,3)$ is also free.

Assume the contrary. Then let $X_{i}$ be an element of (B) such that $\boldsymbol{C} \boldsymbol{s} X_{i}$ is properly contained in $\boldsymbol{C s} A_{i}(i=1,2,3)$. Then (S): $\boldsymbol{C} \boldsymbol{s} X_{i}$ $=n_{5}(i=1,2,3)$.

(3B. 1) $\boldsymbol{C s} A_{i}$ is not nilpotent $(i=1,2,3)$.

Proof. See the proof of (3A. 1).

Now by a theorem of Camina [2] we obtain that $\boldsymbol{C s} A_{i}: \boldsymbol{C s} X_{i}=$ $p_{i}^{a_{i}}$, where $p_{i}$ is a prime, and that $\boldsymbol{Z}\left(\boldsymbol{C} s A_{i}\right)$ is a $p_{i}$-group $(i=1,2,3)$. By the choice of $A_{i}$ the $p_{i}$ are distinct. 
(3B. 2) $\pi\left(\right.$ Cs $\left.A_{i}\right)=\left\{p_{1}, p_{2}, p_{3}\right\} \quad(i=1,2,3)$

Proof. Let $q$ be a prime of $\pi\left(\boldsymbol{C s} A_{1}\right)$ distinct from $p_{i}(i=1,2$, 3). We may assume that $\boldsymbol{C s} A_{1}$ contains a Sylow $q$-subgroup $\mathfrak{Q}$ of (S). Let $Q \neq E$ be an element of $\boldsymbol{Z}(\mathfrak{Q})$. Then we have that $\boldsymbol{C s} A_{1} Q$ contains $\mathfrak{Q}$ and $\left|\boldsymbol{C s} A_{1} Q\right|=\left|\boldsymbol{C} \boldsymbol{s} X_{1}\right|$. This shows that $(\mathcal{S}$ is of isolated type and hence $(B)$ is not simple $[6]$.

(3B. 3) Let $|\boldsymbol{C s} X|=\left|\boldsymbol{C s} X_{1}\right|$. Then $\boldsymbol{C s} X$ is abelian.

Proof. See the proof of (3A. 3)

(3B. 4) We may choose $X=X_{1}$ and $A_{i}(i=1,2,3)$ so that $\boldsymbol{C s} X$ is contained in $\bigcap_{i=1}^{3} \boldsymbol{C s} A_{i}$.

Proof. See the proof of (3A. 4).

Let $\mathfrak{P}_{i}$ be a Sylow $p_{i}$-subgroup of $\boldsymbol{C s} A_{i}$. Then by (3B. 3) $\boldsymbol{C s} A_{i}=$ $\mathfrak{P}_{i} \boldsymbol{C s} X$. In particular, $\boldsymbol{C s} A_{i}$ is solvable $(i=1,2,3)[5$, p. 674]

(3B. 5) $p_{i}=2$ for $i=1$ or 2 or 3 .

Proof. Assume the contrary. Then by a theorem of FeitThompson [3] $\boldsymbol{C s} A_{4}$ is of even order. Since $\boldsymbol{C s} A_{4}$ is free, $\boldsymbol{C s} A_{4}$ is abelian [6]. In particular, a Sylow 2-subgroup of (B) is abelian. Therefore, by a theorem of Walter [13] we get a contradiction.

We assume that $p_{3}=2$. Then $\mathfrak{P}_{3}$ is not abelian and, in particular, of exponent $\geqq 4$.

(3B. 6) There exists a 2-element $Y$ such that $|\boldsymbol{C s} Y|=|\boldsymbol{C s} X|$.

Proof. Assume the contrary. Let $A_{3}^{\prime}$ be an element of $\boldsymbol{Z}\left(\boldsymbol{C s} A_{3}\right)$ of order 4. Let $A$ be any involution of $\boldsymbol{C s} A_{3}$. Then since $\operatorname{Cs} A A_{3}^{\prime}$ is contained in $\boldsymbol{C s}\left(A_{3}^{\prime}\right)^{2}$, we obtain that $\boldsymbol{C s} A_{3}^{\prime} A=\boldsymbol{C} \boldsymbol{s} A=\boldsymbol{C} \boldsymbol{s} A_{3}$. This implies that $\mathfrak{P}_{3}$ is abelian. This is a contradiction.

(3B. 7) We can take $Y$ as in (3B. 4). 
Proof. Since $\boldsymbol{C s} Y$ is minimal, $\boldsymbol{C s} Y$ is the direct product of the Sylow 2-subgroup and the abelian Sylow 2-complement. The rest is obvious.

(3B. 8) $\boldsymbol{F}\left(\boldsymbol{C s} A_{3}\right)$ is not a 2-group.

Proof. Assume the contrary. By a theorem of Fitting [5, p. 277] we have that $\boldsymbol{F}\left(\boldsymbol{C s} A_{3}\right)$ contains $\mathfrak{F}_{3}^{*}$ properly. Let $\mathrm{A}$ be an element of $\boldsymbol{F}\left(\boldsymbol{C} \boldsymbol{s} A_{3}\right) \cap \boldsymbol{N} \boldsymbol{s} \mathfrak{F}_{3}^{*}$ outside $\mathfrak{R}_{3}^{*}$. Then if $A$ belongs to $\boldsymbol{N s} \mathfrak{P}_{1}^{*}$, $\left[A, \mathfrak{R}_{1}^{*}\right]$ is contained in $\mathfrak{P}_{1}^{*} \cap \boldsymbol{F}\left(\boldsymbol{C} \boldsymbol{s} A_{3}\right)=\mathfrak{G}$. Since $\boldsymbol{C s} \mathfrak{P}_{1}^{*} \cap \boldsymbol{C} \boldsymbol{s} A_{3}$ is contained in $\boldsymbol{C s} X$, this is a contradiction. Therefore, $A^{-1} \mathfrak{F}_{1}^{*} A \neq \mathfrak{F}_{1}^{*}$ and $\left[\mathfrak{F}_{3}^{*}, A^{-1} \mathfrak{P}_{1}^{*} A\right]=\mathfrak{F}$. This shows that $|\boldsymbol{C s} X|=\left|\boldsymbol{C} \boldsymbol{s} A_{3}\right|$. This contradicts (3B. 6).

(3B. 9) Let $\left|\boldsymbol{C s} X^{\prime}\right|=|\boldsymbol{C s} X|$. Then $\boldsymbol{C s} X^{\prime}$ is conjugate with Cs $X$ in (S).

Proof. By (3B. 3) $\boldsymbol{C s} X^{\prime}$ is abelian. Since $\boldsymbol{C s} X$ contains $\boldsymbol{Z}\left(\mathfrak{P}_{3}\right)$, we may assume that $\boldsymbol{C s} X$ contains a 2 -element $A_{3}^{\prime}$ of $\boldsymbol{C} \boldsymbol{s} X^{\prime}$. Then $\boldsymbol{C} \boldsymbol{s} A_{3}^{\prime}$ contains both $\boldsymbol{C} \boldsymbol{s} X$ and $\boldsymbol{C} \boldsymbol{s} X^{\prime}$. Now by (3B. 8) $\boldsymbol{F}\left(\boldsymbol{C s} A_{3}^{\prime}\right)$ is not a 2-group. This implies that $\boldsymbol{C s} X=\boldsymbol{C} \boldsymbol{s} X^{\prime}=\boldsymbol{C s}\left(\mathfrak{P}_{1}^{*} \mathfrak{F}_{2}^{*}\right)$.

Now every element of $(S)$ is conjugate either to an element of $\boldsymbol{C s} A_{4}$ or to an element of $\boldsymbol{C s} X$. Since $\boldsymbol{C s} X$ is normal in $\boldsymbol{C s} A_{3}, \boldsymbol{N} \boldsymbol{s}$ $(\boldsymbol{C s} X)$ contains $\boldsymbol{C s} X$ properly. Since $\boldsymbol{C s} A_{4}$ is abelian or an $p$-group of exponent $p$, if $\boldsymbol{N} \boldsymbol{s}\left(\boldsymbol{C s} A_{4}\right)=\boldsymbol{C s} A_{4}$ then by the transfer theorem of Wielandt [5, p. 447] (3) is not simple. Hence $\boldsymbol{N s}\left(\boldsymbol{C s} A_{4}\right) \neq \boldsymbol{C s} A_{4}$. Therefore by counting the number of elements in (S) we get a contradiction.

Part $C$. We use the same notation as in Part $A$. By Parts $A$ and $B$ we may assume that $\boldsymbol{C s} A_{3}$ and $\boldsymbol{C} \boldsymbol{s} A_{4}$ are free. The purpose of this part is to prove that at least one of $\boldsymbol{C s} A_{i}(i=1,2)$ is also free.

Assume the contrary. Then let $X_{i}$ be an element of (B) such that $\boldsymbol{C s} X_{i}$ is properly contained in $\boldsymbol{C s} A_{i}(i=1,2)$. Then (S): $\boldsymbol{C s} X_{i}=$ 
$n_{5}(i=1,2)$.

(3C. 1) $\quad \boldsymbol{C} \boldsymbol{s} A_{i}$ is not nilpotent $(i=1,2)$.

Proof. See the proof of (3A. 1).

Now by a theorem of Camina [2] we obtain that $\boldsymbol{C s} A_{i}: \boldsymbol{C s} X_{i}=$ $p_{i}^{c_{i}}$, where $p_{i}$ is a prime, and that $\boldsymbol{Z}\left(\boldsymbol{C s} A_{i}\right)$ is a $p_{i}$-group $(i=1,2)$. By the choice of $A_{1}$ and $A_{2}, p_{1}$ and $p_{2}$ are distinct.

(3C. 2) $p_{1}$ or $p_{2}=2$.

Proof. See the proof of (3B. 5).

We assume that $p_{2}=2$. Then $P_{2}$ is not abelian, and, in particular, of exponent $\geqq 4$.

(3C. 3) There exists a 2-element $X$ such that $|\boldsymbol{C s} X|=\left|\boldsymbol{C s} X_{1}\right|$. Cs $X$ is the direct product of the Sylow 2-subgsoup $\mathfrak{P}_{2}^{*}$, the abelian Sylow $p_{1}$-subgroup $\mathfrak{H}_{1}^{*}$ and the abelian Hall $\left\{2, p_{1}\right\}$-complement $\mathfrak{A}$ of $\boldsymbol{C s} X$.

Proof. See the proof of (3B. 6).

(3C. 4) We may choose $A_{1}$ and $A_{2}$ so that $\boldsymbol{C s} A_{1} \cap \boldsymbol{C s} A_{2}=\boldsymbol{C s} X$.

Proof. Obvious.

Since $\boldsymbol{C s} A_{i}=\mathfrak{S}_{i} \boldsymbol{C s} X, \boldsymbol{C} \boldsymbol{s} A_{i}$ is solvable $(i=1,2)[\mathbf{5}$, p. 674].

(3C. 5) $\boldsymbol{F}\left(\boldsymbol{C} \boldsymbol{s} A_{2}\right)$ is not a 2-group.

Proof. See the proof of (3B. 8).

Therefore $\boldsymbol{F}\left(\boldsymbol{C s} A_{2}\right)=\boldsymbol{C s} X=\mathfrak{P}_{1}^{*} \times \mathfrak{P}_{2}^{*} \times \mathfrak{A}$. Since $\mathfrak{P}_{2} \boldsymbol{C s} X / \mathfrak{P}_{2}^{*}$ is a Frobenius group with $\boldsymbol{C s} X / \mathfrak{H}_{2}^{*}$ the kernel, $\mathfrak{F}_{2} / \mathfrak{F}_{2}^{*}$ is cyclic or generalized quaternion. Let $A_{2}^{\prime}$ be an element of $\mathfrak{P}_{2}$ outside $\mathfrak{P}_{2}^{*}$. If $\left|\boldsymbol{C s} A_{2}^{\prime}\right|$ $=|\boldsymbol{C s} X|$, then $A_{2}$ commutes with a $p_{1}$-element not belonging to $\mathfrak{P}_{1}^{*}$. This is a contradiction. Hence $\left|\boldsymbol{C s} A_{2}^{\prime}\right|=\mid \boldsymbol{C s} A_{2}^{\prime}$. If $\boldsymbol{C s} A_{2}^{\prime}$ contains a 2-element $X^{\prime}$ of $\boldsymbol{C s} A_{2}$ such that $\boldsymbol{C s} X^{\prime}=\boldsymbol{C} \boldsymbol{s} X$, then $A_{2}^{\prime}$ belongs to 
$\boldsymbol{C s} X$. This is a contradiction. Hence $\boldsymbol{C s} A_{2}^{\prime}$ does not contain such an element. If $\left\langle A_{2}^{\prime}\right\rangle \cap \mathfrak{P}_{2}^{*} \neq \mathfrak{F}$, then $\boldsymbol{C s} A_{2}^{\prime}$ contains $\mathfrak{P}_{1}^{*} \times \mathfrak{A}$. This is a contradiction. Hence $\left\langle A_{2}^{\prime}\right\rangle \cap \Re_{2}^{*}=\mathfrak{F}$.

(3C. 6) $\boldsymbol{Z}\left(\mathfrak{P}_{2}\right)$ is elementary abelian.

Proof. First we show that $\boldsymbol{Z}\left(\boldsymbol{C s} A_{2}\right)$ is elementary abelian. Otherwise, we may assume that $A_{2}$ is an element of order 4 . Let $A_{2}^{\prime}$ be an involution of $\mathfrak{P}_{2}$ outside $\mathfrak{P}_{2}^{*}$. Then $\boldsymbol{C s} A_{2}^{\prime} A_{2}=\boldsymbol{C} s A_{2}^{2}=\boldsymbol{C s} A_{2}$. This shows that $A_{2}^{\prime}$ belongs to $\boldsymbol{Z}\left(\boldsymbol{C s} A_{2}\right)$, and hence to $\boldsymbol{C s} X$. This is a contradiction. Now assume that $\boldsymbol{Z}\left(\mathfrak{P}_{2}\right)$ is not elementary abelian. Let $A_{2}^{\prime \prime}$ be an element of $\boldsymbol{Z}\left(\mathfrak{P}_{2}\right)$ of order 4 . Then $A_{2}^{\prime \prime}$ does not belong to $\mathfrak{P}_{2}^{*}$ by the first argument. But $A_{2}^{\prime \prime} X=X A_{2}^{\prime \prime}$. This is a contradiction.

(3C. 7) $\mathfrak{P}_{2}: \mathfrak{P}_{2}^{*}=2$.

Proof. This is obvious by (3C. 6) and the argument following (3C. 5).

(3C. 8) Let $A_{2}^{\prime}$ be an element of $\mathfrak{F}_{2}$ outside $\mathfrak{P}_{2}^{*}$. Then $\operatorname{Cs} A_{2}^{\prime} \cap$ $\mathfrak{P}_{2}^{*}=\left\langle A_{2}\right\rangle$.

Proof. Let $G$ be an element of (S) such that $\boldsymbol{Z}\left(G^{-1} \mathfrak{P}_{2} G\right)$ contains $A_{2}^{\prime}$. Then $G^{-1} A_{2} G$ belongs to $\boldsymbol{Z}\left(G^{-1} \mathfrak{P}_{2} G\right), \boldsymbol{C s} G^{-1} A_{2} G: \boldsymbol{C s} G^{-1} X G$ $=2$ and $C_{s} G^{-1} X G=G^{-1} \mathfrak{P}_{1}^{*} G \times G^{-1} \mathfrak{F}_{2}^{*} G \times G^{-1} \mathfrak{X} G$. Then $A_{2}^{\prime}$ belongs to $G^{-1} \mathfrak{P}_{2}^{*} G$. Hence $\boldsymbol{C} \boldsymbol{s} A_{2}^{\prime}=\boldsymbol{C} \boldsymbol{s} G^{-1} A_{2} G$. Now assume that $\boldsymbol{C s} A_{2}^{\prime} \cap \mathfrak{P}_{2}^{*}$ contains $\left\langle A_{2}\right\rangle$ properly. Then $\boldsymbol{C s} G^{-1} X G$ contains an element $A_{2}^{\prime \prime} \neq E$ of $\mathfrak{P}_{2}^{*}$. Then $\boldsymbol{C s} A_{2}^{\prime \prime}$ contains $\mathfrak{P}_{1}^{*}$ and $G^{-1} \mathfrak{B}_{1}^{*} G$. The first argument shows that $\boldsymbol{F}\left(\boldsymbol{C} \boldsymbol{s} A_{2}^{\prime \prime}\right)$ is not a 2-group. Hence $\mathfrak{P}_{1}^{*}=G^{-1} \mathfrak{P}_{1}^{*} G$. This is a contradiction.

Now by a lemma of Suzuki [11] $\mathfrak{P}_{2}$ is dihedral or quasi-dihedral. Hence by a theorem of Gorenstein-Walter [4] or a theorem of AlperinBrauer-Gorenstein [1] we get a contradiction.

Remark. The argument in $((c)$, p. 244) of [8] is incomplete, since the argument appeals to [9] which is not applicable in that 
case. One way to amend it is to follow the argument in Part $C$.

Part $D$. We use the same notation as in Part $A$. By Parts $A$, $B$ and $C$ we may assume that $C \boldsymbol{s} A_{i}(i=1,2,3,4)$ is free. The purpose of this part is to prove that $(S)$ is isomorphic with some $S z(l)$, where $l=2^{2 n+1}, n \geqq 1$, or $L F(3,4)$. By [7] $\boldsymbol{C s} A_{1}$ is not free. Let $X$ be an element of $\mathbb{B}$ such that $\boldsymbol{C s} A_{1}: \boldsymbol{C s} X=n_{5}$.

(3D. 1) $C s A_{1}$ is a Hall subgroup of $\mathbb{S}$. Furtheremore, $|\mathbb{S}|=$ $\prod_{i=1}^{4}\left|\boldsymbol{C} \boldsymbol{s} A_{i}\right|$.

Proof. This is obvious.

(3D. 2) $\boldsymbol{C s} A_{1}$ is of even order.

Proof. See the proof of (3B. 5).

(3D. 3) We may assume that $C_{s} A_{1}$ is not nilpotent.

Proof. If $\boldsymbol{C s} A_{1}$ is nilpotent, then by a theorem of Wielandt [5, p. 285] all subgroups $\mathfrak{X}$ of $(3)$ with $|\mathfrak{X}|=\left|\boldsymbol{C} s A_{1}\right|$ are nilpotent. Hence, in particular, the centralizer of every involution of $(B)$ is 2 closed. Therefore by a theorem of Suzuki [12] we get the theorem. Hence we may assume that $\boldsymbol{C s} A_{1}$ is not nilpotent.

Now by a theorem of Camina $[2]$ we obtain that $\boldsymbol{C} \boldsymbol{s} A_{1}: C \boldsymbol{s} X=$ $p^{a}$, where $p$ is a prime, and that $\boldsymbol{Z}\left(\boldsymbol{C s} A_{1}\right)$ is a $p$-group.

(3D. 4) We may assume that $p=2$.

Proof. Otherwise, let $J$ be an involution in $C \boldsymbol{s} A_{1}$. Then $\boldsymbol{C s} J=$ Cs $A_{1} J$ is nilpotent. Hence, as in the proof of (3D. 3) we may assume that $p=2$.

Now, as before, $\mathfrak{B}_{1}$ is not abelian and, in particular, of exponent $\geqq 4$.

(3D. 5) There exists a 2-element $Y$ such that $|\boldsymbol{C s} Y|=|\boldsymbol{C s} X|$. 
Proof. See the proof of (3B. 6).

(3D. 6) $\boldsymbol{F}\left(\boldsymbol{C s} A_{1}\right)$ is not a 2-group.

Proof. Since $\boldsymbol{C s} A_{1}=\mathfrak{F}_{1} \boldsymbol{C s} Y$ and since $\boldsymbol{C s} Y$ is nilpotent, $\boldsymbol{C s} A_{1}$ is solvable [5, p. 674]. Now see the proof of (3B. 8).

By (3D. 3) $\left|\pi\left(\boldsymbol{C s} A_{1}\right)\right| \geqq 2$. Let $q$ be a prime of $\pi\left(\boldsymbol{C s} A_{1}\right)$ distinct from 2. Let $\mathfrak{Q}$ be a Sylow $q$-subgroup of $\boldsymbol{C s} A_{1}$. Let $X \neq E$ be an element of $\boldsymbol{Z}(\mathfrak{Q})$. Then $\boldsymbol{C} \boldsymbol{s} X=\boldsymbol{C} \boldsymbol{s} X A_{1}$ is the direct product of the abelian Sylow 2-subgroup $\mathfrak{P}_{1}^{*}$, the Sylow $q$-subgroup $\mathfrak{D}$ and the abelian Hall $\{2, q\}$-complement $\mathfrak{A}$. By (3D. 5) $\mathfrak{Q}$ is also abelian. Thus Cs $X$ is abelian.

Now to complete the proof it suffices to prove the following proposition, which is incompatible with (3D. 6)

(3D. 7) $\boldsymbol{F}\left(\boldsymbol{C s} A_{1}\right)$ is a 2-group.

Proof. Assume the contrary. Then $\boldsymbol{F}\left(\boldsymbol{C s} A_{1}\right)=\boldsymbol{C s} X . \mathfrak{P}_{1} \boldsymbol{C s} X / \mathfrak{P}_{1}^{*}$ is a Frobenius group with $\boldsymbol{C s} X / \mathfrak{P}_{1}^{*}$ the kernel. Hence $\mathfrak{P}_{1} / \mathfrak{P}_{1}^{*}$ is cyclic or generalized quaternion [5, p. 502].

First we assume that $\mathfrak{P}_{1} / \mathfrak{P}_{1}^{*}$ is a generalized quaternion group of order $2^{b}$. Then there exist elements $Q$ and $R$ of $\mathfrak{F}_{1}$ and $S, T, U$ and $V$ of $\mathfrak{B}_{1}^{*}$ such that $R^{-1} Q R=Q^{-1} S, Q^{2^{b-2}}=R^{2} T, Q^{2^{b-1}}=U, R^{4}=V$ and $\mathfrak{P}_{1} / \mathfrak{P}_{1}^{*}=\langle Q, R\rangle \mathfrak{P}_{1}^{*} / \mathfrak{P}_{1}^{*}$. Now we further assume that $\mathfrak{P}_{1}^{*}$ is not cyclic. Let $\mathfrak{W}$ be a normal subgroup of $\mathfrak{F}_{1}$ of type $(2,2)$ contained in $\mathfrak{P}_{1}^{*}$. Then $\boldsymbol{C s} R^{2}$ contains $\mathfrak{W}$. If $\left|\boldsymbol{C s} R^{2}\right|=|\boldsymbol{C s} X|$, then by the remark just before (3D. 7) $\boldsymbol{C} s R^{2}$ is abelian. This implies that $\boldsymbol{C} s R^{2}$ is contained in $\boldsymbol{C s} A_{1}$. This is a contradiction. Hence $\left|\boldsymbol{C s} R^{2}\right|=\left|\boldsymbol{C s} A_{1}\right|$. If $\boldsymbol{F}\left(\boldsymbol{C s} R^{2}\right)$ is a 2 -group, then we have that $\mathfrak{F}_{1}: \mathfrak{P}_{1}^{*}>|\mathfrak{\Omega}|$. This is a contradiction. Hence $\boldsymbol{F}\left(\boldsymbol{C s} R^{2}\right)$ is not a 2-group. Let $\mathfrak{F}_{1}, \mathfrak{F}_{1}^{\#}, \mathfrak{Q}^{*}$ and $\mathfrak{A}^{\#}$ be a Sylow 2-subgroup of $\boldsymbol{C s} R^{2}$, the abelian Sylow 2-subgroup, the abelian Sylow $q$-subgroup and the abclian Hall $\{2, q\}$-complement of $\boldsymbol{F}\left(\boldsymbol{C s} R^{2}\right)$, respectively. Then $\mathfrak{F}_{1} / \mathfrak{W}_{\mathbb{*}}^{*}$ is cyclic or generalized quaternion.. This implies that $\mathfrak{W} \cap \mathfrak{P}_{1}^{\sharp} \neq \mathfrak{F}$. Take an element $W \neq E$ of 
$\mathfrak{W} \cap \mathfrak{P}_{1}^{\sharp}$. Then $\boldsymbol{C} \boldsymbol{s} W$ contains $\mathfrak{Q}^{\sharp}$ and $\mathfrak{Q}$. This implies that $\mathfrak{Q}^{\sharp}=\mathfrak{Q}$. This is a contradiction. Therefore $\mathfrak{P}_{1}^{*}$ is cyclic. Hence $\mathfrak{F}_{1} \cap \boldsymbol{C} \mathfrak{s}_{1}^{*}$ contains $\mathfrak{B}_{1}^{*}$ properly. Thus $\boldsymbol{C s} Q^{2^{b-2}}$ contains $\mathfrak{B}_{1}^{*}$. This implies that $\left|\boldsymbol{C} \boldsymbol{s} Q^{2^{b-2}}\right|=\left|\boldsymbol{C} \boldsymbol{s} A_{1}\right|$. As above, $\boldsymbol{F}\left(\boldsymbol{C s} Q^{2^{b-2}}\right)$ is not a 2-group. If $|\boldsymbol{C s} Q|$ $=|\boldsymbol{C} \boldsymbol{s} X|$, then $\boldsymbol{C} \boldsymbol{s} Q=\boldsymbol{F}\left(\boldsymbol{C s} Q^{2^{b-2}}\right)$. Let $\mathfrak{\beta}_{1}$ be the Sylow 2-subgroup of $C s Q$. Then $\left[Q, \mathfrak{P}_{1}^{*}\right]$ is contained in $\mathfrak{P}_{1}^{*} \cap \mathfrak{P}_{1}=(\xi$. This is a contradiction. Hence $\boldsymbol{C} s Q=C s Q^{2^{b-2}}$. Similarly we obtain that $\boldsymbol{C} s R=C s R^{2}$. Since $Q^{2^{b-2}}$ and $R^{2}$ commute, this implies that $Q$ and $R$ commute. This is a contradiction. Therefore $\mathfrak{\beta}_{1} / \mathfrak{P}_{1}^{*}$ is cyclic.

Let $\mathfrak{P}_{1} / \mathfrak{P}_{1}^{*}$ be of order $2^{b}$ and $P \mathfrak{P}_{1}^{*}$ a generator of $\mathfrak{F}_{1} / \mathfrak{P}_{1}^{*}$. Assume that $b \geqq 2$. As above, we may assume that $\mathfrak{P}_{1}$ is cyclic. Therefore $\mathfrak{P}_{1}$ is metacyclic. Then by a theorem of Mazurov [10] $\mathfrak{P}_{1}$ is of type $(2,2)$ or of maximal class. This is a contradiction. Hence we obtain that $b=1$. Now we show that $\boldsymbol{Z}\left(\mathfrak{P}_{1}\right)$ is of order 2. Assume the contrary. If $|\boldsymbol{C} \boldsymbol{s} P|=|\boldsymbol{C} \boldsymbol{s} X|$, then by the remark just before (3D. 7) $\boldsymbol{C s} P$ is abelian and $\boldsymbol{C s} P \cap(\mathfrak{Q} \times \mathfrak{X})=\mathfrak{F}$. Let $\mathfrak{Q}^{\#}$ be the abelian Sylow $q$-subgroup of $\boldsymbol{C s} P$. Then $\mathfrak{Q}^{\sharp} \cap \mathfrak{Q}=\mathfrak{r}$. But since $\boldsymbol{C s} P$ contains $\boldsymbol{Z}\left(\mathfrak{P}_{1}\right)$, this is a contradiction. If $|\boldsymbol{C s} P|=\left|\boldsymbol{C s} A_{1}\right|$, then let $\mathfrak{P}_{1}^{\#}$ arıd $\mathfrak{Q}^{\#}$ be the abelian Sylow 2 -subgroup and the abelian Sylow $q$-subgroup of $\boldsymbol{F}(\boldsymbol{C s} P)$. Then $\mathfrak{P}_{1} \cap \mathfrak{P}_{1}^{\sharp} \neq(5$ by assumption. Let $Z \neq E$ be an element of $\mathfrak{P}_{1} \cap \mathfrak{F}_{1}^{\sharp}$. Cs $Z$ contains $\mathfrak{Z}$ and $\mathfrak{Q}^{\#}$. Since $\mathfrak{Q} \cap \mathfrak{Q}^{\#}=\mathfrak{F}$ and since $\boldsymbol{F}(\boldsymbol{C} \boldsymbol{s} Z)$ contains $\mathfrak{Q}$ and $\mathfrak{Q}^{\sharp}$, this is a contradiction. Hence $\left|\boldsymbol{Z}\left(\mathfrak{P}_{1}\right)\right|=2$. Since $P$ is of order 2 (See the proof of (3C. 6)), by a lemma of Suzuki [11] $\mathfrak{P}_{1}$ is of type $(2,2)$ or of maximal class. Then by a theorem of Wong [14] we get a contradiction.

Remark. The argument of Part $D$, together with [8], shows that we obtain the followiog theorem. Let (S) be a simple group such that $C$ (S) has the following shape $n_{1} n_{2} \cdots n_{k}$. Then $k=3$ or $\grave{n}_{k+1}$

4. If $k=3$, then (8) is isomorphic with $\operatorname{LF}(2, l)$, where $l$ is an odd prime power bigger than 5. If $k=4$, then (S) is isomorphic with $S z(l)$, where $l=2^{2 n+1}, n \geqq 1$, or $\operatorname{LF}(3,4)$. 
Department of Mathematics, University of Illinois Chicago Circle

\section{References}

[1] J.L. Alperin, R. Brauer and D. Gorenstein, Finite groups with quasi-dihedral and wreathed Sylow 2-subgroups, Trans. Am. Math. Soc. 151 (1970), 1-261.

[2] A. R. Camina, Conjugacy classes of groups and some theorems of N. Ito, J. London Math. Soc., to appear.

[3] W. Feit and J. G. Thompson. Solvability of groups of odd order, Pacific J. Math. 13 (1963), 755-1029.

[4] D. Gorenstein and J. H. Walter, The characterization of finite groups with dihedral Sylow 2-groups, J. Algebra 2 (1965), 85-151, 218-270, 354-393.

[5] B. Huppert. "Endliche Gruppen, I", Springer. Berlin/New York, 1967.

[6] N. Ito, On finite groups with given conjugate type I, Nagoya Math. J. 6 (1953), 17-28.

[7] N. Ito, On finite groups with given conjugate type III, Math. Z. 117 (1970), 267-271.

[8] N. Ito, Simple groups of conjugate type rank 4, J. Algebra 20 (1972), 226-249.

[9] N. Ito, A theorem on factorizable groups, Acta Sci. Math. Szeged 33 (1972), 49-52.

[10] V.D. Mazurov, O konečnyh gruppah c metacikliceskimi silovskimi 2-podgruppami, Sibirskiı̌ Math. Ž. 8 (1967). 966-982.

[11] M. Suzuki, A characterization of simple groups LF (2, p), J. Fac. Sci. Univ. Tokyo 6 (1951), 259-293.

[12] M. Suzuki, Finite groups in which the centralizer of any element of order 2 is 2-closed, Ann. of Math. 82 (1965), 191-212.

[13] J.H. Walter, The characterization of finite groups with abelian Sylow 2 subgroups, Ann. of Math. 89 (1969), 405-514.

[14] W. Wong, On finite groups whose 2-Sylow subgroups have cyclic subgroups of index 2, J. Austral. Math. Soc. 4 (1964), 90-112. 